\title{
A new quasilinear formulation for ICRF plasmas in a toroidal geometry
}

\author{
Jungpyo Lee ${ }^{l, *}$, John Wright ${ }^{1}$, Nicola Bertelli ${ }^{2}$, David Smithe $^{3}$, Ernest Valeo ${ }^{2}$, Yuri Petrov ${ }^{4}$, Erwin F. Jaeger ${ }^{5}$, Lee Berry $^{5}$, \\ Robert Harvey ${ }^{4}$, and Paul Bonoli ${ }^{1}$ \\ ${ }^{1}$ MIT Plasma Science and Fusion Center, Cambridge, MA, 02139, USA \\ ${ }^{2}$ Princeton Plasma Physics Laboratory, Princeton, NJ, 08540, USA \\ ${ }^{3}$ Tech-X, Boulder, CO, 80303, USA \\ ${ }^{4}$ CompX, Del Mar, CA, 92014, USA \\ ${ }^{5}$ XCEL Engineering, Oak Ridge, TN, 37830, USA
}

\begin{abstract}
We present a new formulation for quasilinear velocity space diffusion for ICRF plasmas that considers two different aspects: (1) finite Larmor radius approximation and (2) includes the effect of toroidal geometry and constructs a positive definite form. In the first aspect, the Kennel-Engelmann (K-E) quasilinear diffusion coefficients are successfully approximated in a small Larmor radius limit and implemented for the numerical codes (TORIC-CQL3D). In the second aspect, the quasilinear diffusion is reformulated in a toroidal geometry in order to include the parallel dynamics in the inhomogeneous plasmas and magnetic fields. We use these two quasilinear formulations to simulate ITER plasmas with ICRF injection for minority fundamental heating and Tritium second harmonic cyclotron heating.
\end{abstract}

\section{Introduction}

The ion cyclotron range of frequency (ICRF) waves transfer their energy and momentum to plasmas, and as a result a significant amount of fast ions are produced. The kinetic description of the non-Maxwellian distribution plasmas is obtained using quasilinear diffusion coefficients due to the RF waves in a Fokker-Planck equation. The quasilinear diffusion coefficients are derived by Kennel and Engelmann (K-E) [1] with the assumptions of the homogenous plasmas and magnetic fields along the particle trajectory. In this proceeding, we present the theoretical modifications of the K-E diffusion coefficients in two different aspects.

In the first aspect, the coefficients are modified to be consistent with the dielectric tensor of the reduced model in the small Larmor radius approximation. In the reduced model, the dielectric tensor for the plasma current is expanded by a small parameter $k_{\perp} \rho_{i}$, in which $k_{\perp}$ can be replaced by the differential operator [2]. Accordingly, the computation time of the reduced model can be significantly reduced by a factor of $\mathrm{O}\left(n_{r}^{2}\right)$ compared to the full model [3] that uses $k_{\perp}$ explicitly without the small Larmor radius approximation. Here, $n_{r}$ is the number of radial coordinate grid. In Section 2, we summarize the derivation of the equivalent quasilinear diffusion to the reduced model as in our recent publication [4], and present an additional example using the implementation in TORIC-CQL3D.

TORIC is already coupled with a sophisticated Fokker-Planck module, SSFPQL [5, 6]. It is worth noting the different features between our approach in TORIC-CQL3D [4] and that in TORIC-SSFPQL $[5,6]$. CQL3D is the Fokker-Planck code, which has been validated against demonstrated by many $R F$ wave experiments. It has fully nonlinear and relativistic collision operators and it can calculate the time evolution of distribution function for the tail formation and relaxation by transient RF waves. For the quasilinear diffusion, our formulation in TORIC-CQL3D guarantees the consistency with the dielectric tensor, while it cannot include the contribution from the higher order in $k_{\perp} \rho_{i}$ that is retained in the K-E coefficients used in TORICSSFPQL. As the result of the self-consistency, the power absorptions by dielectric tensor and quasilinear diffusion theoretically match each other and the numerical iterations between the Maxwell's equation solver and the Fokker-Planck code likely converge without any normalization factor to the diffusion coefficients [4].

In the second aspect, we modify the formulation of K-E coefficients to be positive-definite in the toroidal geometry. The positive definite form can include some important features of the diffusion in the toroidal geometry which cannot be captured in the K-E coefficients, as will be explained in Section 3. Using this new positive-definite from, we can eliminate the unphysical growing mode violating $\mathrm{H}$-theorem and the related numerical errors in the coupled code TORICCQL3D. To best of our knowledge, it is the first implementation of the positive-definite form for the quasilinear diffusion coefficient in a continuum FokkerPlanck solver for a toroidal geometry.

\section{FLR approximations}

\subsection{Kinetic energy change $(\dot{W})$}

The rate of the kinetic energy change due to the RF waves $(\dot{W})$ is used to define the quasiliner diffusion coefficients in a specific numerical formulation. For example, in the full model using a full Fourier spectral $\boldsymbol{k}$

\footnotetext{
Corresponding author: Jungpyo@psfc.mit.edu
} 
space, the K-E quasilinear diffusion coefficients can be defined by

$$
\begin{aligned}
\dot{W}= & \frac{1}{2} \operatorname{Re}\left[\sum_{\boldsymbol{k}_{\mathbf{1}}} \sum_{\boldsymbol{k}_{\mathbf{2}}} e^{i\left(\boldsymbol{k}_{\mathbf{1}}-\boldsymbol{k}_{2}\right) \cdot \boldsymbol{r}}\left(\boldsymbol{E}_{\boldsymbol{k}_{\mathbf{1}}} \cdot \mathbf{W}_{\boldsymbol{l}} \cdot \boldsymbol{E}_{\boldsymbol{k}_{\mathbf{2}}}\right)\right] \\
& =\int \boldsymbol{d} \boldsymbol{v} \frac{m v^{2}}{2} Q(f)
\end{aligned}
$$

where $\mathbf{W}_{\boldsymbol{l}}$ is the resonance Kernel (e.g. Eq. (11) of [3]). For energy transfer, the quasilinear diffusion coefficients are gyroaveraged, and they can be described in the velocity space of speed-pitchangle coordinate $(v, \vartheta)$ by

$$
\begin{gathered}
Q(f)=\frac{1}{v^{2}} \frac{\partial}{\partial v}\left\{B \frac{\partial}{\partial v}+C \frac{\partial}{\partial \vartheta}\right\} f \\
+\frac{1}{v^{2} \operatorname{SIN} \vartheta} \frac{\partial}{\partial \vartheta}\left\{E \frac{\partial}{\partial v}+F \frac{\partial}{\partial \vartheta}\right\} f .
\end{gathered}
$$

The relation between the coefficients $B, C, E$, and $F$ are given to preserve the diffusion directions in velocity space [6].

In the reduced model, the kinetic energy can be defined to be consistent with the approximation of plasma currents using the expansion of the gyromotion around the gyrocenter. For example, when evaluating $\dot{W}$, the electric field at the gyrocenter is approximated by

$$
\begin{gathered}
E\left(r_{g}\right)=\left[1-\left(r-r_{g}\right) \cdot \nabla+\frac{1}{2}\left(r-r_{g}\right)(r-\right. \\
\left.\left.r_{g}\right): \nabla \nabla\right] E(r),
\end{gathered}
$$

and it results in the expansion of $\dot{W}$ in terms of the small parameters $k_{\perp} \rho_{i}$,

$$
\dot{W}=\dot{W}^{(0)}+\dot{W}^{(1)}+\dot{W}^{(2)}+\cdots,
$$

where the superscript indicates the order of $k_{\perp} \rho_{i}$. The detailed derivations for Eq. (4) are in Section 2 of [4].

\subsection{Quasilinear diffusion coefficient}

The K-E quasilinear diffusion coefficient corresponding to $\dot{W}$ in the full model of Eq. (1) is

$$
\begin{gathered}
B=\frac{\pi \epsilon w_{p}}{2 m n_{s}} \sum_{n} \operatorname{Re}\left[\sum_{\boldsymbol{k}_{\mathbf{2}}} v_{\perp} \chi_{\boldsymbol{k}_{2}, \boldsymbol{n}}^{*} e^{-i \boldsymbol{k}_{2} \cdot \boldsymbol{r}}\right. \\
\left.\sum_{\boldsymbol{k}_{\mathbf{1}}} v_{\perp} \chi_{\boldsymbol{k}_{\mathbf{1}}, \boldsymbol{n}} e^{i \boldsymbol{k}_{\mathbf{1}} \cdot \boldsymbol{r}} \delta\left(\omega-n \Omega-k_{\|, 1} v_{\|}\right)\right]
\end{gathered}
$$

where $n$ is the integer to determine the harmonic number of ion cyclotron resonance and $\chi_{\boldsymbol{k}}$ is the effective potential [3].

To derive the quasilinear diffusion coefficients corresponding to $\dot{W}$ in the reduced model of Eq. (4), three important properties of the diffusion need to be considered: positive-definiteness, wave polarization, and, diffusion direction. We found a way to formulate the coefficients to preserve these three properties [4]. The coefficient $B$ is formulated from the lowest order of $\dot{W}$, and other coefficients are calculated from the original relations with $B$.

Accordingly, for the fundamental damping $(\mathbf{n}=1)$, the coefficient is defined by $\dot{W}^{(0)}$, giving

$$
\begin{gathered}
B^{(n=1)}=\frac{\pi \epsilon w_{p}}{2 m n_{S}} \operatorname{Re}\left[\sum_{\boldsymbol{k}_{2}} v_{\perp} E_{+\boldsymbol{k}_{2}, \boldsymbol{n}}^{*} e^{-i \boldsymbol{k}_{2} \cdot \boldsymbol{r}}\right. \\
\left.\sum_{\boldsymbol{k}_{\mathbf{1}}} v_{\perp} E_{+\boldsymbol{k}_{\mathbf{1}}, \boldsymbol{n}} e^{i \boldsymbol{k}_{\mathbf{1}} \cdot \boldsymbol{r}} \delta\left(\omega-n \Omega-k_{\|, 1} v_{\|}\right)\right] .
\end{gathered}
$$

For the second harmonic damping $(n=2)$, the coefficient is defined by $\dot{W}^{(2)}$, giving

$$
\begin{array}{r}
B^{(n=2)}=\frac{\pi \epsilon 2 w_{p}^{2}}{8 m n_{s}} \operatorname{Re}\left[\frac{v_{\perp}^{2}}{\Omega^{2}} \sum_{\boldsymbol{k}_{\mathbf{2}}} v_{\perp} E_{+\boldsymbol{k}_{2}, \boldsymbol{n}}^{*} e^{-i \boldsymbol{k}_{2} \cdot \boldsymbol{r}}\right. \\
\left.\partial_{-}\left(\sum_{\boldsymbol{k}_{\mathbf{1}}} v_{\perp} \partial_{+} E_{+\boldsymbol{k}_{1}, \boldsymbol{n}} e^{i \boldsymbol{k}_{1} \cdot \boldsymbol{r}} \delta\left(\omega-n \Omega-k_{\|, 1} v_{\|}\right)\right)\right],
\end{array}
$$

where the detail derivation can be found in [4]

\subsection{Examples}

The quasilinear diffusion coeffcients for the reduced model are evaluated in the wave code by the reduced model, TORIC [2]. Using the diffusion coefficients, TORIC is coupled with a Fokker-Planck code CQL3D [6], to have the self-consistent solutions of distribution and wave fields. In the recent paper [4], we simulate the scenario for ITER 10MW He3 minority species damping $(n=1)$ by the reduced model TORIC-CQL3D, and compared the results with the full model by AORSACQL3D [3]. The comparisons show that the reduced model is acceptably valid when the considerable amount of fast ions satisfies $k_{\perp} \rho_{i} \preccurlyeq 1.0$ and the wave power density is less than $1 \mathrm{MW} / \mathrm{m}^{3}$ in the scenario.

In this proceeding, we introduce the results of the second harmonic damping $(n=2)$ scenarios of ITER 5.3T. We simulate two ion species with the ratio of $(\mathrm{D}, \mathrm{T})=$ $(50,50) \%$, and the ICRF wave frequency is $52.5 \mathrm{MHz}$. The dominant wave power is absorbed by the second harmonic damping of the major species Tritium around the magnetic axis because $\omega=2 \Omega$ at $\mathrm{R}=\mathrm{R}_{0}+0.17 \mathrm{~m}$. The power decompositions are reasonably similar between TORIC and AORSA: for Maxwellian plasmas, $49 \%$ of T second harmonic damping and $48 \%$ electron damping in TORIC, while $48 \%$ of $\mathrm{T}$ second harmonic damping, and $51 \%$ electron damping in AORSA. For self-consistent non-Maxwellian plasmas, $48 \%$ of $\mathrm{T}$ second harmonic damping, and $46 \%$ electron damping in TORIC, while $61 \%$ of $\mathrm{T}$ second harmonic damping, and $39 \%$ electron damping in AORSA.

Figure 1 and 2 show the distribution functions of TORIC-CQL3D and AORSA-CQL3D, which show the similar patterns but some difference in the tails around $v_{\|} \simeq 0$. The main reason of difference could be the error of the approximation on the Bessel function factor $J_{1}\left(k_{\perp} \rho_{i}\right) \simeq k_{\perp} \rho_{i} / 2$ for the high energetic Tritium of several MeV. The error of the small Larmor radius approximation can be larger for $n=2$ than that of $n=1$ in [4]. 
(a)

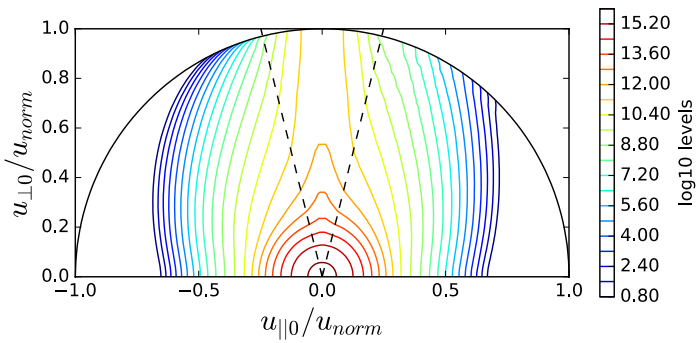

(b)

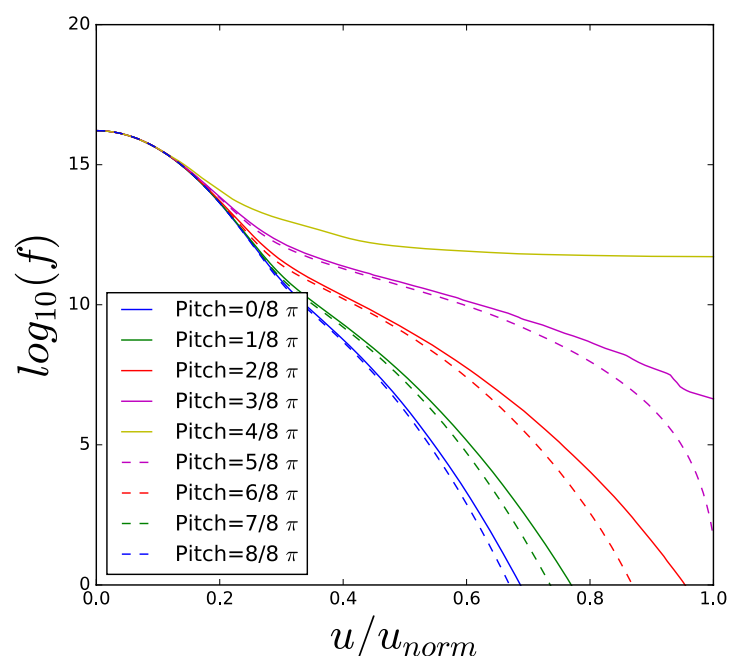

Fig. 1. Reduced model results by TORIC-CQL3D : (a) 2-D contour plots of the distribution function in velocity space at $\mathrm{r} / \mathrm{a}=0.1$ (b) 1-D distribution functions in terms of speed for several pitch-angles. Here, unorm is the momentum corresponding to the energy of Tritium $4 \mathrm{MeV}$.

(a)

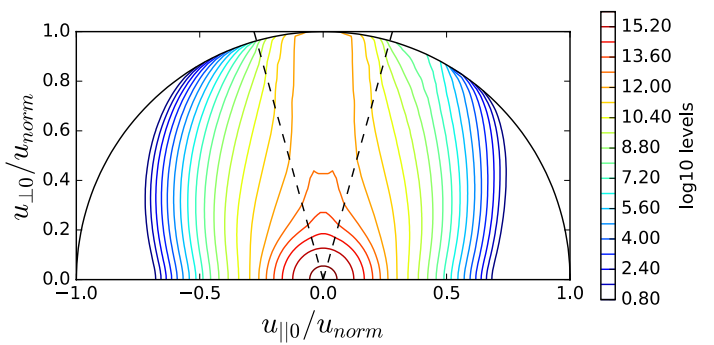

(b)

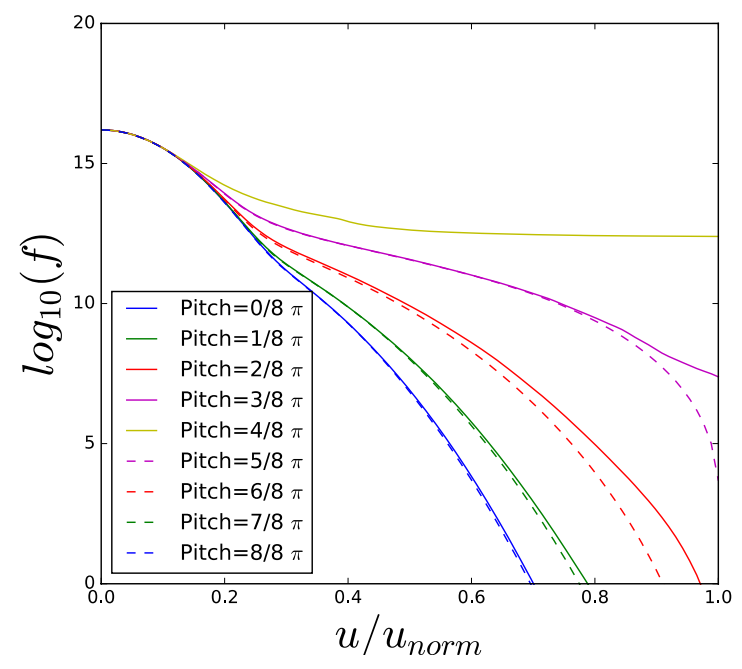

Fig. 2. Full model results by AORSA-CQL3D : (a) 2-D contour plots of the distribution function in velocity space at $\mathrm{r} / \mathrm{a}=0.1$ (b) 1-D distribution functions in terms of speed for several pitch-angles.

\section{Toroidal geometry effect}

\subsection{Positive definite form}

In a toroidal geometry, the magnetic field is inhomogeneous along the particle trajectory and the assumption of K-E coefficients is violated. The violation is typically ignorable because of the small correlation length of the wave-plasma interaction compared to the variation of the magnetic fields. However, we found that it does not result in the positive-definiteness when the quasilinear diffusion coefficients are bounce-averaged for the bounce-averaged Fokker-Planck code, CQL3D [6]. As a consequence, the negative values in the bounce-averaged diffusion coefficient $\langle B\rangle_{b}=\int d l B / v_{\|}$ cause unphysical growing modes and numerical errors when enforcing to eliminate them.

The positive-definiteness of the bounce-averaged coefficients are recovered when treating the trajectory integral and the bounce integral as the same way in the toroidal geometry [7]. For example, the bounce-averaged coefficient is reformulated by

$$
\begin{gathered}
\langle B\rangle_{b}=\frac{\pi \epsilon w_{p}}{2 m n_{s}} \int_{-\infty}^{0} d t \operatorname{Re}\left[\sum_{\boldsymbol{k}_{2}}\left(\ldots \boldsymbol{E}_{\boldsymbol{k}_{2}}\right) e^{-i \boldsymbol{k}_{\mathbf{2}} \cdot \boldsymbol{r}(\boldsymbol{t})}\right. \\
\left.\left\langle\sum_{\boldsymbol{k}_{\mathbf{1}}} \int_{0}^{\infty} d \tau e^{i 2 \gamma t} e^{i \omega \tau}\left(\ldots \boldsymbol{E}_{\boldsymbol{k}_{\mathbf{1}}}\right) e^{-i \boldsymbol{k}_{\mathbf{1}} \cdot \boldsymbol{r}(\boldsymbol{t}-\boldsymbol{\tau})}\right\rangle_{\boldsymbol{g}}\right] \\
=\frac{\pi \epsilon w_{p}}{2 m n_{s}} \operatorname{Re}\left[\sum_{\boldsymbol{n}} \sum_{\boldsymbol{k}_{\mathbf{2}}} \sum_{\boldsymbol{k}_{\mathbf{1}}} E_{+\boldsymbol{k}_{\mathbf{2}}, \boldsymbol{n}}^{*} A\left(\boldsymbol{k}_{\mathbf{1}}, \boldsymbol{k}_{\mathbf{2}}\right) E_{+\boldsymbol{k}_{\mathbf{1}}, \boldsymbol{n}}\right],
\end{gathered}
$$

where $\langle\ldots\rangle_{g}$ is the gyroaverage and the symmetric operator $A\left(\boldsymbol{k}_{1}, \boldsymbol{k}_{2}\right)=A^{*}\left(\boldsymbol{k}_{\mathbf{2}}, \boldsymbol{k}_{\mathbf{1}}\right)$ is obtained by the change of variables $t_{1}=t-\tau$ and $t_{2}=t$, giving

$$
A\left(\boldsymbol{k}_{1}, \boldsymbol{k}_{2}\right)=\frac{1}{2} \int_{-\infty}^{0} d t_{1} \int_{-\infty}^{0} d t_{2} v_{\perp}\left(t_{1}\right)
$$$$
v_{\perp}\left(t_{2}\right) e^{i \Phi_{n}\left(t_{2}, k_{\|, 2}\right)-i \Phi_{n}\left(t_{1}, k_{\|, 1}\right)} \text {, }
$$

and it guarantees the positive-definiteness in the toroidal geometry. In Eq. (5), K-E coefficients have the Diracdelta function depending on only $k_{\|, 1}$ because of the 
uniform phase only in the trajectory integral, and it breaks the symmetry shown in Eq. (9).

\subsection{Trajectory integral}

The evaluation of the trajectory integral in Eq. (9) is not trivial because of the phase variation in the toroidal geometry,

$$
\frac{\partial \Phi_{n}}{\partial t}=\omega-n \Omega(t)-k_{\|}(t) v_{\|}(t)
$$

The stationary approximation around the resonance point $\partial \Phi_{n} / \partial t=0$ is useful for the short correlation length. By expanding $\Phi_{n}$ in terms of the small temporal distance from the resonance $t-t_{r}$ up to third order, it results in the Airy functions $(A i)$, as many previous studies investigated $[8,9]$,

$$
\begin{gathered}
\int_{-\infty}^{0} d t v_{\perp}(t) e^{i \Phi_{n}\left(t, k_{\|}\right)} \simeq v_{\perp}\left(t_{r}\right) e^{i \Phi_{n}\left(t_{r}, k_{\|}\right)} R\left(t_{r}\right) \\
R\left(t_{r}\right)=\frac{\pi}{\alpha^{\frac{1}{3}}} e^{-i \alpha\left(x_{1} t_{+}+\frac{t_{+}^{3}}{3}\right)} A i\left(\alpha^{\frac{3}{2}} x_{1}\right)
\end{gathered}
$$

where $\alpha=\left|0.5 \partial^{3} \Phi_{n} / \partial t^{3}\right|, t_{+}=\left|\partial^{2} \Phi_{n} / \partial t^{2} / \partial^{3} \Phi_{n}\right|$ $\partial t^{3} \mid$, and $x_{1}=-t_{+}^{2}$ at $t=t_{r}$, result in the negative argument of the Airy function.

When $\partial^{2} \Phi_{n} / \partial t^{2}=0$ is satisfied, the correlation length becomes large so that the variation of the phase could be important even for the non-resonance locations with $\partial \Phi_{n} / \partial t \neq 0$. This condition typically occurs at the inner-midplane and outer-midplane for passing particles and trapping tips and outer-midplane for trapped particles [9]. We can include this non-resonant contribution to the diffusion by the positive arguments of the Airy function,

$$
\begin{gathered}
\int_{-\infty}^{\infty} d t v_{\perp}(t) e^{i \Phi_{n}\left(t, k_{\|}\right)} \simeq v_{\perp}\left(t_{n r}\right) e^{i \Phi_{n}\left(t_{n r}, k_{\|}\right)} N\left(t_{n r}\right)(13) \\
N\left(t_{n r}\right)=\frac{\pi}{\alpha^{\frac{1}{3}}}\left(A i\left(\alpha^{\frac{3}{2}} x_{2}\right)+A i\left(\alpha^{\frac{3}{2}} x_{2}\right)^{*}\right)
\end{gathered}
$$

where $x_{2}=2\left|\partial \Phi_{n} / \partial t / \partial^{3} \Phi_{n} / \partial t^{3}\right|$ at the specific conditions $t=t_{n r}$.

The resonant contribution in Eq. (11) and the nonresonant contribution in Eq. (13) are smoothly connected by the Airy function, and it results in the continuous diffusion coefficients in velocity space.

\subsection{Implementation}

This positive definite form of the bounce-averaged coefficients in Eq. (8) is implemented in TORIC. The resonant contribution is evaluated when the spectral mode has at least one resonance location on a flux surface, and the non-resonant contribution is included when the spectral mode has no resonant location.

The phase integral $\Phi_{n}$ is not poloidally periodic on a general flux surface with the parallel spectral mode number $(m+n q)$ not being an integer. Thus, the evaluation of the diffusion coefficient depends on the initial position of the integral range, and the average diffusion changes depending on the number of poloidal periods in the evaluations. We will show the importance of the initial position and evaluation range in a future publication.

Additionally, the positive definite form can show the correlation between the consecutive resonance crossings, while the kicks by the Dirac-delta function of the K-E diffusion coefficients are likely randomized. This correlation between resonances are important in the two consecutive resonance around the location of $t=t_{n r} \quad$ (e.g. outer-midplane), where the toroidal geometry effect needs to be considered within the relatively long correlation length.

This new quasilinear diffusion is expected to be beneficial for ICRF modelling in a low aspect ratio tokamak that has the significantly varying magnetic field along particle trajectories. In spite of the good features to capture the toroidal effects in the quasilinear diffusion, this positive definite form is computationally expensive because it requires about $n_{v} n_{m} n_{c h} n_{\text {int }} \sim 10^{6}$ times more number of floating-point operations than evaluating K-E quasilinear diffusion in Eq. (5). Here, $n_{v} \sim 100, n_{m} \sim 100, n_{c h} \sim 10$, and $n_{\text {int }} \sim 10$ are the number of velocity space grid in each dimension, the number of polodal spectral grid, the order of interpolation, the required number of interpolation, respectively.

This work was supported by US DoE Contract No. DE-FC0201ER54648 under a Scientific Discovery through Advanced Computing Initiative.

\section{References}

1. C.F. Kennel, and F. Engelmann Phys. Fluids 9, 2377 (1966)

2. M. Brambilla, Plasma Physics and Controlled Fusion 41, 1 (1999).

3. E. F. Jaeger, L. A. Berry, E. DAzevedo, D. B. Batchelor, and M. D. Carter, Physics of Plasmas 8, 1573 (2001).

4. J. P. Lee, J. C. Wright, N. Berterlli, E. F. Jaeger, E. Valeo, R. W. Harvey, and P. T. Bonoli, Phys. Plasmas 24, 052502 (2017)

5. M. Brambilla and R. Bilato, Nuclear Fusion 46, S387-S396 (2006).

6. M. Brambilla and R. Bilato, Nuclear Fusion 49, 085004 (2009).

7. R. W. Harvey, and M. G. McCoy, in Proc. IAEATCM on Advances in Sim. and Modeling of Thermonuclear Plasmas, available through USDOC/NTIS No. DE93002962 (1992) , pp. 489526

8. D. Smithe, Plasma Physics and Controlled Fusion 31, 1105 (1989)

9. I.B. Bernstein and D.C. Baxter, Phys. Fluids 24, 108 (1981)

10. P.J. Catto and J.R. Myra, Phys. Fluids B 4,187 (1992) 